\title{
New Modalities of Cycloablation and High-Intensity-Focused Ultrasound
}

\author{
Natasha Nayak Kolomeyer and Marlene R. Moster
}

\subsection{Introduction}

Cycloablative or cyclodestructive procedures aim to lower intraocular pressure (IOP) by decreasing the function of the ciliary body and thereby decreasing the rate of aqueous production. Cycloablative procedures were typically used in refractory glaucoma in eyes with poor visual potential; however, more focused energy and targeted destruction of the ciliary body has led to an increase in cyclodestructive treatment options that are now an important adjunct to our surgical armamentarium.

\subsection{Transscleral Diode Cyclophotocoagulation (TSCPC)}

Cyclodestructive procedures have evolved since the 1920s, progressing from cyclectomy, cyclodiathermy, cyclocryotherapy, and eventually to cyclophotocoagulation [1-3]. Cyclophotocoagulation was initially performed in 1961 by using light from xenon arc photocoagulation [4] and subsequently using a ruby laser in 1969 [5]. Cyclophotocoagulation established a more widespread application once Nd:YAG (neodymium-yttrium-aluminum garnet) and eventually semiconductor diode lasers were developed. Nd:YAG cyclophotocoagulation (1064 nm wavelength) can be performed with or without ocular surface contact; however, noncontact methods were relinquished due to their higher complication rates. Currently, semiconductor diode lasers are the mainstay of transscleral diode cyclophotocoagulation (TSCPC). They have several advantages including greater uveal melanin absorption, compact size, and minimal maintenance requirements compared to prior lasers. Human cadaveric studies demonstrate epithelial coagulative necrosis and thermal coagulation of the ciliary stroma and vasculature in eyes receiving diode TSCPC [6].

N. N. Kolomeyer $(\bowtie) \cdot$ M. R. Moster

Glaucoma Department, Wills Eye Hospital, Philadelphia, PA, USA

(C) The Author(s) 2021

C. C. A. Sng, K. Barton (eds.), Minimally Invasive Glaucoma Surgery,

https://doi.org/10.1007/978-981-15-5632-6_9 


\subsubsection{Procedure}

- Typically, local anesthesia or sedation is administered along with regional (retrobulbar/peribulbar/subtenon's) anesthesia.

- Common diode laser settings: Duration is often set at 2-3 s. Power starts at $1250-2500 \mathrm{~mW}$. The power is titrated in $250 \mathrm{~mW}$ increments (maximum $\sim 4000 \mathrm{~mW}$ ) until an audible "pop" is heard; the power is then titrated to $250 \mathrm{~mW}$ below the "pop" threshold [3].

- The number of spot treatments varies between 14 and 20, sparing the 3 and 9 o'clock in order to avoid the long posterior ciliary nerves and vessels.

- The G-Probe is placed just posterior to the limbus, perpendicular to the limbus. This places the fiberoptic $1.2 \mathrm{~mm}$ posterior to the limbus. Maintain gentle pressure on the G-Probe throughout the treatment duration.

- Some physicians choose to administer a subconjunctival injection of steroids after TSCPC. The eye is patched and a shield is placed over the eye.

- Patients are placed on steroid drops after the laser, with the frequency of topical steroids titrated according to the severity of inflammation. All preoperative glaucoma medications are continued in the immediate postoperative period and can be selectively stopped depending on the IOP response. Patients are typically seen 1 day and 1 week postoperatively and subsequently depending on patient response.

\subsubsection{Indications}

TSCPC is indicated in patients with refractory glaucoma or a blind painful eye. It is typically used in patients with poor visual acuity (VA). It can also be used earlier in patients where incisional surgery is less ideal, such as those with significant medical conditions, bleeding diathesis, or scarred conjunctiva. The laser power for TSCPC may be reduced when treating eyes with good vision to reduce the risk of sightthreatening complications.

\subsubsection{Results}

A thorough compilation of the results and complications of TSCPC can be referred to in a recent review article; we have highlighted the most relevant findings below [7]. The treatment effect is often seen around 1 month after TSCPC; it is advisable to wait at least 1 month for retreatment if possible. TSCPC has demonstrated a range of effect on IOP (12.3-66\% IOP reduction); post-laser IOP of $\leq 21 \mathrm{mmHg}$ has been reported in $54-92.7 \%$ of eyes. Various studies suggest a correlation between IOP reduction and the amount of energy per treatment session or the number of laser burns. However, there are several studies that could not find a direct correlation. Other factors that influence treatment success include pre-laser IOP and the subtype of glaucoma. Lower success rates have been reported in aphakic, traumatic, and juvenile glaucoma. TSCPC success rates also increase with age and decrease with a 
history of prior surgery. More pigmented eyes usually require less energy, but there is no clear relationship with TSCPC success rates.

\subsubsection{Complications}

Adverse effects associated with treatment include vision loss (8.8-47\%), hypotony (0-26\%), hyphema (0-2\%), anterior uveitis (9-28\%), pupillary changes $(0.8-50 \%)$, phthisis $(0-10 \%)$, retinal detachment $(1 \%)$, IOP spike, cataract progression, vitreous hemorrhage, lens subluxation, necrotizing scleritis, and rarely sympathetic ophthalmia [1].

The literature suggests a relationship between the amount of energy delivered and the risk of hypotony and phthisis [8]. It is unclear if this is a nonlinear relationship, but treatment sessions utilizing more than $80 \mathrm{~J}$ of energy tend to have higher rates of these complications. Glaucoma subtype (including neovascular glaucoma [NVG]) and high pretreatment IOP are also considered risk factors for hypotony and phthisis. This suggests that lower energy settings in a high-risk patient may be important in minimizing these complications.

Loss of more than two Snellen lines of VA was reported on average in $22.5 \%$ of eyes (0-55\% range) [7]. Rotchford et al. evaluated the outcomes of TSCPC in patients with VA of at least 20/60. After 5 years, $73.5 \%$ of patients had an IOP of $16 \mathrm{mmHg}$ or less and $30.6 \%$ had lost two or more Snellen lines of VA [9]. The proportion of patients who lost vision is consistent with that reported after incisional surgery, suggesting that TSCPC can be considered as an option in selected eyes with good visual potential.

Concerns regarding postoperative complications must be balanced against overall efficacy for each individual patient, as studies suggest a relationship between the amount of laser energy delivered and IOP reduction, as well as the risk of hypotony and phthisis.

\subsection{Micropulse Transscleral Cyclophotocoagulation (MP-TSCPC)}

The micropulse delivery mode of diode laser (MP-TSCPC, MicroPulse P3, IRIDEX IQ810 Laser System, Mountain View, CA, USA) is a more recent form of TSCPC. MP-TSCPC operates in an "on" and "off" cycle mode, delivering $810 \mathrm{~nm}$ infrared radiation from a diode source. During the "on" cycle, multiple bursts of laser are emitted by the device resulting in an increase in thermal energy absorption in pigmented tissues and induction of coagulative necrosis. Theoretically, the nonpigmented tissues do not cross the coagulative threshold because they have a lower rate of thermal energy absorption and are able to cool off during the "off" cycle. The MP-TSCPC also employs a novel probe that: (a) allows sweeping, continuous applications compared to individual spot treatments and (b) targets the pars plana rather than the pars plicata. 


\subsubsection{Procedure}

- MP-TSCPC can be performed under topical, regional (peribulbar/subtenon's/retrobulbar), or general anesthesia. Some physicians find that topical or local anesthesia with a short burst of heavy sedation can be used without a need for retrobulbar anesthesia when in the operating room. However, if done in an office setting, regional (retrobulbar/peribulbar/subtenon's) anesthesia is commonly employed.

- Default laser settings are: micropulse mode, $2000 \mathrm{~mW}$ power, duty cycle of $31.33 \%$, micropulse "on" time of $0.5 \mathrm{~ms}$, and micropulse "off" time of $1.1 \mathrm{~ms}$. At the surgeon's discretion, the laser is delivered over $360^{\circ}$ for $100-360 \mathrm{~s}$, while sparing the 3 and 9 o'clock positions as above for TSCPC. The duration of treatment is often titrated based on the patient's history. While the default laser settings are $2000 \mathrm{~mW}$, more recently surgeons have titrated the power settings in the 2000-2500 mW range based on the patient's history as well.

- The MP-TSCPC probe is placed along the limbus perpendicular to the sclera. The probe is then moved in a continuous, sliding, slow motion around the limbus, sparing 3 and 9 o'clock, while applying firm pressure. The rate of movement is encouraged to be around 10-20 s per quadrant. The probe tip is designed to position the fiberoptic tip $3 \mathrm{~mm}$ posterior to the limbus (Fig. 9.1).

- Some physicians choose to administer a subconjunctival injection of steroids after MP-TSCPC.

- Topical steroid drops are typically applied four times daily post-surgery to control the inflammation and subsequently tapered as inflammatory response decreases.
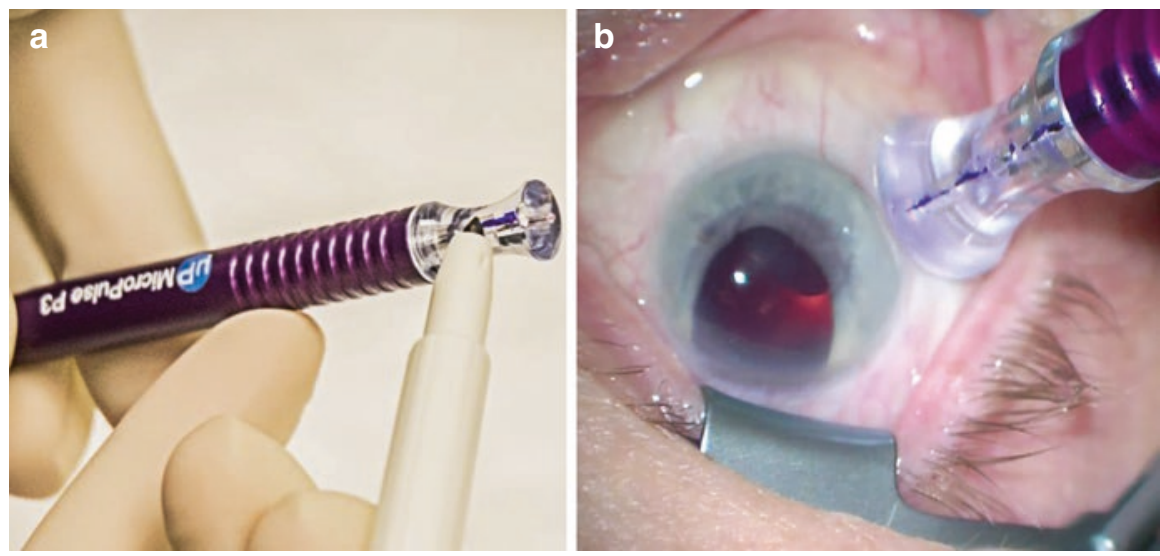

Fig. 9.1 Micropulse transscleral cyclophotocoagulation (MP-TSCPC). (a) The notch on the probe is to be placed toward the limbus. The notch is located on the rounder half of the probe and can be marked for easier visibility with a marker. (b) The probe is then placed with the marked notch perpendicular to the limbus. (Copyright Marlene Moster, MD; Bill Romano; and Natasha Nayak Kolomeyer, MD; reproduced with permission) 


\subsubsection{Indications}

Indications for MP-TSCPC are broad, spanning noninvasive early interventions as well as refractory primary and secondary glaucomas. We recommend reviewing possible complications with the patient if using MP-TSCPC as an earlier intervention.

\subsubsection{MP-TSCPC vs. TSCPC}

MP-TSCPC versus TSCPC: A randomized exploratory study compared results of MP-TSCPC (with $100 \mathrm{~s}$ treatment duration) and continuous TSCPC in 48 patients with refractory end-stage glaucoma [10]. A successful primary outcome measure (IOP 6-21 mmHg and at least a 30\% reduction with or without antiglaucoma medications after 18 months) was achieved after MP-TSCPC and continuous TSCPC in $52 \%$ and $30 \%(p=0.13)$, respectively. There was a significant difference at 1 year (75\% vs. $29 \%)$ that reached statistical significance $(p<0.01)$. Mean IOP was reduced by $45 \%$ in both groups from a baseline of 36.5 and $35 \mathrm{mmHg}$ after $17.5 \pm 1.6$ months of follow-up. There was no significant difference in the number of IOP-lowering medications, while the complication rate was higher in the continuous TSCPC $(p=0.01)$ group including prolonged anterior chamber $(\mathrm{A} / \mathrm{C})$ inflammation, hypotony, and phthisis bulbi. There was a greater degree of IOP variance in the continuous TSCPC group, but the treatment settings were also more variable.

\subsubsection{Results}

Tan et al. [11] conducted a prospective case series of 40 eyes with refractory glaucoma that received a mean of 1.4 treatments of $100 \mathrm{~s}$ of MP-TSCPC. Eighty percent of eyes achieved relative success (IOP $<21 \mathrm{mmHg}$ or reduction of $30 \%$ from baseline) with or without supplemental glaucoma medication, with $65 \%$ of eyes achieving successful IOP after one treatment. The average follow-up period of $17.3 \pm 2.0$ months was significantly longer than most other studies.

A retrospective review of 79 refractory glaucoma patients who received 120-360 s of MP-TSCPC demonstrated a treatment success rate of $75 \%$ at 3 months (IOP of 6-21 mmHg or a reduction of IOP by 20\%), with an additional $10 \%$ of patients meeting the success criteria after the addition of IOP-lowering medications [12]. At 6 months, the treatment success rates dropped to $66 \%$ and were stable until the last follow-up for patients with at least 6 months of follow-up.

Emanuel et al. conducted a retrospective review of 84 eyes with a mean follow up of 4.3 months. There was a $41 \%$ and $53 \%$ reduction in IOP at 1 month and 3 months postoperatively, respectively [13].

Another retrospective review compared $320 \mathrm{~s}$ of MP-TSCPC results in nine pediatric (age 1-17 years) and 37 adult glaucoma patients [14]. At the 12-month followup period, success was achieved in $72 \%(26 / 36)$ of adult patients but only in $22 \%$ 
(2/9) of pediatric patients $(p=0.02)$. Success was defined as IOP between 5 and $21 \mathrm{mmHg}$ and $\geq 20 \%$ reduction from baseline at 12 months of follow-up without the use of oral carbonic anhydrase inhibitors, loss of light perception vision, or reoperation for glaucoma within the 12-month follow-up period. The mean IOP at postoperative months 1 and 6 was significantly decreased from baseline in the pediatric group, but the effect lost significance at 12 months. A majority (7/9) of pediatric eyes required reoperation to control IOP during the follow-up period.

\subsubsection{Complications}

The complications of MP-TSCPC in the retrospective review of 79 eyes by Williams et al. [12] included 7 patients with hypotony (9\%), 21 (26\%) patients with prolonged A/C inflammation, $13(16 \%)$ patients with loss of two or more lines of bestcorrected VA for $\geq 3$ months, $4(5 \%)$ patients with macular edema, $2(2.5 \%)$ patients with corneal edema, and $2(2.5 \%)$ patients with phthisis bulbi. There were no reported cases of mydriasis or loss of accommodation; however, given the retrospective nature of the study, this information was not directly elicited from the patients. The ten patients who underwent re-treatment did not seem to be more inclined to complications.

Tan et al. [11] did not observe any cases of hypotony or loss of vision after MP-TSCPC. All eyes had mild postoperative inflammation that resolved by 2 weeks in $90 \%$ of eyes and by 4 weeks in the remainder of eyes. Seven (17.5\%) eyes with NVG developed hyphema. This study employed shorter treatment duration (100 s) compared to Williams et al. (120-360 s). Further studies would be required to ascertain whether there is a treatment duration related effect on outcomes and complications.

Emanuel et al. [13] observed $5(6 \%)$ cases of persistent hypotony, 3 (4\%) cases of IOP spikes, as well as hyphema (4\%) and choroidals (1\%). Persistent inflammation at postoperative month 3 was found in $74 \%$ of eyes. At postoperative month 1 , $35 \%$ of eyes lost two more lines of vision. Three patients lost light-perception vision but were light perception at baseline. Tan et al. also found that MP-TSCPC caused significantly greater conjunctival inflammation and scarring compared to controls in Dutch Belted Rabbits, similar to continuous wave TSCPC [25]. Hence, further studies are required to investigate the effect of post-TSCPC conjunctival changes on future bleb morphology and survival.

\subsection{High-Intensity Focused Ultrasound (HIFU)}

High-Intensity Focused Ultrasound (HIFU) (Therapeutic Ultrasound System; Sonocare Inc., Ridgewood, NJ, USA) was evaluated as an alternative to ciliary body destruction in the 1980s [15]. Interest in HIFU initially faded due to the duration and complexity of treatment as well as significant complications (scleral staphyloma, phthisis, persistent hypotony, corneal thinning, and vision loss). However, modifications of the HIFU technology have resulted in recent renewed interest in this treatment modality. A miniaturized HIFU technique, ultrasonic circular cyclophotocoagulation (UC3, EyeOP1 
HIFU, EyeTechCare, Rillieux-la-Pape, France), employs a circular operator-independent probe that focuses the ultrasound energy circumferentially on the ciliary body without operator movement. Unlike diode laser, focused ultrasound technology can treat a defined tissue volume at any depth or location within the eye, without being affected by pigmentation. The complex transducers and higher operating frequency allow for more selective treatment areas.

\subsubsection{Procedure}

- UC3 can be performed under topical, regional (peribulbar/subtenon's/retrobulbar), or general anesthesia.

- The coupling cone is placed in direct contact with the ocular surface and centered around the limbus. The coupling cone is then connected to a suction ring that establishes a low-level vacuum $(70 \mathrm{mmHg})$ to maintain the cone in contact with the ocular surface to achieve alignment and control distance during the procedure. The probe is inserted into a coupling cone; probes are available in 11, 12, and $13 \mathrm{~mm}$ ring diameters (ring diameter size is determined preoperatively based on biometric data). The space between the ocular surface, the coupling cone, and the probe is filled with about $4 \mathrm{~mL}$ of room-temperature balanced salted solution. The probe (30 mm diameter, $15 \mathrm{~mm}$ height) is divided into six cylindrical piezoceramic transducers that generate ultrasound beams that allow treatment of up to $45 \%$ of the ciliary body. The ultrasound beam is focused at the depth of the ciliary body (2 mm below the sclera) [1] (Fig. 9.2).

- Each of the six transducers is activated for 4, 6, or $8 \mathrm{~s}$ (depending on treatment protocol), with a $20 \mathrm{~s}$ gap between each transducer, allowing cool down between each partial treatment. The entire treatment, which automatically proceeds with the activated foot pedal, is about $2.5 \mathrm{~min}$.

- The settings specifically aim to avoid treatment of the retina, cornea, lens, as well as nasal and temporal zones.
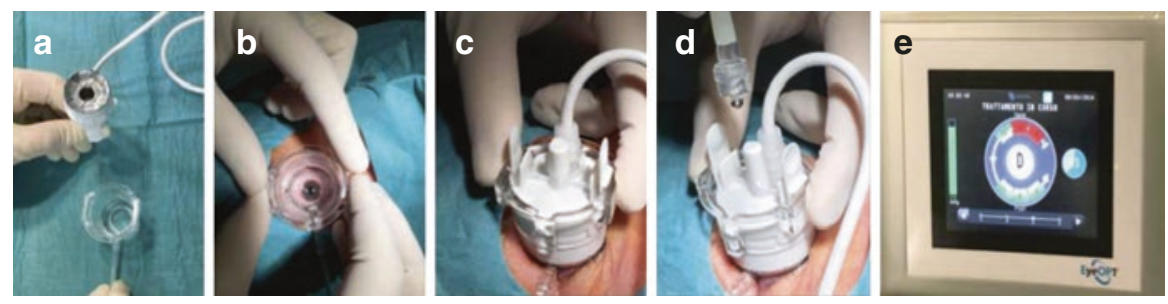

Fig. 9.2 Ultrasonic circular cyclophotocoagulation (UC3) procedure comprises two elements: the probe with the six piezoelectric transducers generating the ultrasound beam and the coupling cone (a). The correctly positioned cone must show a homogeneous ring of visible sclera; when this ring is regular, the cone is then maintained by a mild vacuum system (b). After verification of the effective suction, the probe is inserted and stabilized into the cone (c). During the procedure, the cone is continuously filled with saline solution (d), in order to allow the ultrasound transmission. The treatment starts in the superior sectors with a progressive activation of each transducer (e). (This figure and description have been reproduced from an open-access article by Mastropasqua et al. [1]) 


\subsubsection{Mechanism}

Ultrasound can cause thermal increase of up to $80{ }^{\circ} \mathrm{C}$. The primary mechanism of action is reduction of aqueous production due to thermal necrosis of the ciliary epithelium. Histopathology studies in rabbits by Aptel et al. demonstrated focal necrotic changes in distal and intermediate ciliary processes, while sparing the basal and remaining parts of the ciliary body [15]. Untreated adjacent areas lacked signs of inflammation and had preserved architecture and vasculature. Additionally, there appears to be a correlation of treatment dosage and extent of ciliary process destruction [1]. Additional mechanisms of action could include modification of the scleral and conjunctival anatomy [16] and an increase in suprachoroidal and transscleral outflow [17-19].

\subsubsection{Results}

Giannacare et al. conducted a prospective multicenter interventional study of 30 eyes on maximum medical therapy with a 6-month follow-up [20]. Qualified and complete success (IOP reduction $\geq 20 \%$ and IOP $\geq 5 \mathrm{mmHg}$ ) was achieved in $70 \%$ and $46.7 \%$ of patients, respectively, while treatment failure was recorded in $6.6 \%$. Eyes that were randomized to receive greater ultrasound exposure time $(8 \mathrm{~s}$ per transducer) had greater IOP reduction compared to eyes with shorter ultrasound exposure time (4 or $6 \mathrm{~s}$ ). There was significant IOP reduction on postoperative day $1(39 \%)$ despite study protocol requiring discontinuation of ocular hypotensive medications in the immediate postoperative period unless IOP was above $21 \mathrm{mmHg}$.

The EyeMUST1 study was a prospective multicenter interventional study of 52 patients with refractory glaucoma [18]. Patients received either $4 \mathrm{~s}$ (group 1) or $6 \mathrm{~s}$ (group 2) exposure time per transducer (non-randomized). Success was defined as at least a $20 \%$ IOP reduction and IOP $>5 \mathrm{mmHg}$ without additional hypotensive medications but with possible HIFU retreatment. Success was achieved at 6 months in $61.9 \%$ of group 1 patients and $65.4 \%$ of group 2 patients, while at 12 months the proportion was $57.1 \%$ and $48.0 \%$, respectively (no significance at either time point). This difference was not statistically significant. Eight (15\%) patients received HIFU retreatment. Twelve (22\%) patients required a secondary glaucoma surgical intervention between 6 and 12 months post-HIFU.

Melamed et al. performed a prospective interventional study of 20 patients who received HIFU treatment (using 6 s exposure time per transducer), with $4(20 \%)$ requiring retreatment [21]. Complete (IOP reduction $\geq 20 \%$ and IOP $>5 \mathrm{mmHg}$ ) and qualified (allowance of additional medication and/or retreatment) surgical success was achieved in $45 \%$ and $65 \%$, respectively.

Aptel et al. conducted a multicenter prospective clinical trial of 30 glaucoma patients without prior history of filtering surgery with a $6 \mathrm{~s}$ exposure time per transducer and 12-month follow up [22]. Complete success (IOP reduction $\geq 20 \%$, IOP $>5 \mathrm{mmHg}$ and IOP $<21 \mathrm{mmHg}$ with possible re-intervention and without additional hypotensive medication) was achieved in $47 \%$ of eyes and qualified success (allowance of retreatment without additional medications) was found in $63 \%$ of eyes. 
De Gregorio et al. completed a prospective interventional study of 40 patients with an $8 \mathrm{~s}$ exposure time per transducer [23]. At 4 months, if the IOP was $>21 \mathrm{mmHg}$ with no adverse major complications related to HIFU procedure, the decision was made to retreat. Success was defined as IOP $>5 \mathrm{mmHg}$ and $\mathrm{IOP}<21 \mathrm{mmHg}$ without hypotensive medication and vision-threatening complications. Eighteen (45\%) eyes achieved complete success with a mean IOP reduction of $44.3 \%$ at 4 months and $45.7 \%$ at 12 months. At 12 months, success was achieved in $85 \%$ of treated eyes with a maximum of three HIFU procedures.

\subsubsection{Complications}

Transient complications such as a fixed and dilated pupil (0-3\%), anterior chamber inflammation (20-24\%), superficial punctate keratitis (13-45\%), subconjunctival hemorrhage (4-30\%), corneal edema (7-20\%), IOP spikes (0-7\%), induced corneal astigmatism ( $0-3 \%)$, macular edema $(0-3 \%)$, and hypotony $(0-2 \%)$ have been identified [18, 20-23]. Loss of three or more lines of best-corrected VA was reported in $5-20 \%$ of patients $[18,22]$. Notably, DeGregorio et al. noted scleral thinning in the treated sectors in $25 \%$ of eyes [23]. This evidence along with AS-OCT data suggesting scleral remodeling after $\mathrm{UC}_{3}$ treatment highlights the importance of further analysis of the degree of scleral remodeling and how this may affect future filtration surgery. To our knowledge, there are no reports of persistent hypotony or phthisis after $\mathrm{UC}_{3}$ treatment.

It is important to note that the $\mathrm{UC}_{3}$ device requires suction to couple the device to the ocular surface, which increases the IOP for a period of $2.5 \mathrm{~min}$. Although there are no reported cases of associated optic neuropathy, vein/artery occlusion, or visual field loss related to this specific device, this should be considered given the reports of LASIK-related complications [24].

\subsection{Conclusion}

Cycloablative treatment options for glaucoma continue to evolve. Recent developments such as MP-TSCPC and HIFU have improved safety profiles with variable results. We encourage the readers to balance the importance of safety and efficacy when choosing any surgical procedure, but especially a cycloablative procedure.

\section{References}

1. Mastropasqua R, Fasanella V, Mastropasqua A, Ciancaglini M, Agnifili L. High-intensity focused ultrasound circular cyclocoagulation in glaucoma: a step forward for cyclodestruction? J Ophthalmol. 2017;2017:7136275. Pubmed Central PMCID: 5420440

2. Pantcheva MB, Schuman JS. Chandler and Grant's glaucoma. 5th ed. Thorafore, NJ: SLACK Incorporated; 2013. p. 511-22.

3. Rand Allingham R, et al. Shields textbook of glaucoma. 6th ed. Philadelphia, PA: Lippincott Williams \& Wilkins; 2011. 
4. Weekers R, Lavergne G, Watillon M, Gilson M, Legros AM. Effects of photocoagulation of ciliary body upon ocular tension. Am J Ophthalmol. 1961;52:156-63.

5. Vucicevic ZM, Tsou KC, Nazarian IH, Scheie HG, Burns WP. A cytochemical approach to the laser coagulation of the ciliary body. Bibl Ophthalmol. 1969;79:467-78.

6. Schuman JS, Noecker RJ, Puliafito CA, Jacobson JJ, Shepps GJ, Wang N. Energy levels and probe placement in contact transscleral semiconductor diode laser cyclophotocoagulation in human cadaver eyes. Arch Ophthalmol. 1991;109(11):1534-8.

7. Ishida K. Update on results and complications of cyclophotocoagulation. Curr Opin Ophthalmol. 2013;24(2):102-10.

8. Vernon SA, Koppens JM, Menon GJ, Negi AK. Diode laser cycloablation in adult glaucoma: long-term results of a standard protocol and review of current literature. Clin Exp Ophthalmol. 2006;34(5):411-20.

9. Rotchford AP, Jayasawal R, Madhusudhan S, Ho S, King AJ, Vernon SA. Transscleral diode laser cycloablation in patients with good vision. Br J Ophthalmol. 2010;94(9):1180-3.

10. Aquino MC, Barton K, Tan AM, Sng C, Li X, Loon SC, et al. Micropulse versus continuous wave transscleral diode cyclophotocoagulation in refractory glaucoma: a randomized exploratory study. Clin Exp Ophthalmol. 2015;43(1):40-6.

11. Tan AM, Chockalingam M, Aquino MC, Lim ZI, See JL, Chew PT. Micropulse transscleral diode laser cyclophotocoagulation in the treatment of refractory glaucoma. Clin Exp Ophthalmol. 2010;38(3):266-72.

12. Williams AL, Moster MR, Rahmatnejad K, Resende AF, Horan T, Reynolds M, et al. Clinical efficacy and safety profile of micropulse transscleral cyclophotocoagulation in refractory glaucoma. J Glaucoma. 2018;27:445-9.

13. Emanuel ME, Grover DS, Fellman RL, Godfrey DG, Smith O, Butler MR, et al. Micropulse cyclophotocoagulation: initial results in refractory glaucoma. J Glaucoma. 2017;26(8):726-9.

14. Lee JH, Shi Y, Amoozgar B, Aderman C, De Alba CA, Lin S, et al. Outcome of micropulse laser transscleral cyclophotocoagulation on pediatric versus adult glaucoma patients. J Glaucoma. 2017;26(10):936-9.

15. Coleman DJ, Lizzi FL, Driller J, Rosado AL, Burgess SE, Torpey JH, et al. Therapeutic ultrasound in the treatment of glaucoma. II. Clinical applications. Ophthalmology. 1985 Mar;92(3):347-53.

16. Mastropasqua R, Agnifili L, Fasanella V, Toto L, Brescia L, Di Staso S, et al. Uveo-scleral outflow pathways after ultrasonic cyclocoagulation in refractory glaucoma: an anterior segment optical coherence tomography and in vivo confocal study. Br J Ophthalmol. 2016;100(12):1668-75.

17. Aptel F, Charrel T, Lafon C, Romano F, Chapelon JY, Blumen-Ohana E, et al. Miniaturized high-intensity focused ultrasound device in patients with glaucoma: a clinical pilot study. Invest Ophthalmol Vis Sci. 2011;52(12):8747-53.

18. Denis P, Aptel F, Rouland JF, Nordmann JP, Lachkar Y, Renard JP, et al. Cyclocoagulation of the ciliary bodies by high-intensity focused ultrasound: a 12-month multicenter study. Invest Ophthalmol Vis Sci. 2015;56(2):1089-96.

19. Aptel F, Dupuy C, Rouland JF. Treatment of refractory open-angle glaucoma using ultrasonic circular cyclocoagulation: a prospective case series. Curr Med Res Opin. 2014;30(8):1599-605.

20. Giannaccare G, Vagge A, Gizzi C, Bagnis A, Sebastiani S, Del Noce C, et al. High-intensity focused ultrasound treatment in patients with refractory glaucoma. Graefes Arch Clin Exp. 2017;255(3):599-605.

21. Melamed S, Goldenfeld M, Cotlear D, Skaat A, Moroz I. High-intensity focused ultrasound treatment in refractory glaucoma patients: results at 1 year of prospective clinical study. Eur J Ophthalmol. 2015;25(6):483-9.

22. Aptel F, Denis P, Rouland JF, Renard JP, Bron A. Multicenter clinical trial of high-intensity focused ultrasound treatment in glaucoma patients without previous filtering surgery. Acta Ophthalmol. 2016;94(5):e268-77. 
23. De Gregorio A, Pedrotti E, Stevan G, Montali M, Morselli S. Safety and efficacy of multiple cyclocoagulation of ciliary bodies by high-intensity focused ultrasound in patients with glaucoma. Graefes Arch Clin Exp. 2017;255(12):2429-35.

24. Cameron BD, Saffra NA, Strominger MB. Laser in situ keratomileusis-induced optic neuropathy. Ophthalmology. 2001;108(4):660-5.

25. Tan NYQ, Ang M, Chan ASY, et al. Transscleral cyclophotocoauglation and its histological effects on the conjunctiva. Sci Rep. 2019;9(1):18703.

Open Access This chapter is licensed under the terms of the Creative Commons Attribution 4.0 International License (http://creativecommons.org/licenses/by/4.0/), which permits use, sharing, adaptation, distribution and reproduction in any medium or format, as long as you give appropriate credit to the original author(s) and the source, provide a link to the Creative Commons license and indicate if changes were made.

The images or other third party material in this chapter are included in the chapter's Creative Commons license, unless indicated otherwise in a credit line to the material. If material is not included in the chapter's Creative Commons license and your intended use is not permitted by statutory regulation or exceeds the permitted use, you will need to obtain permission directly from the copyright holder.

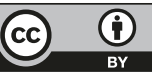

